\title{
La construcción social de la confianza en investigación cualitativa. El caso de la Vega Alta del Segura (Región de Murcia)
}

\author{
The social building of trust in the qualitative research. The case \\ of Vega Alta del Segura (Region of Murcia)
}

\author{
Antonio José Ramírez-Melgarejo \\ Universidad de Murcia \\ ajrm1@um.es (ESPAÑA)
}

Recibido: 18.072018
Aceptado: 18.03 .2020

\section{RESUMEN}

Los municipios de Abarán, Blanca y Cieza conforman un importante enclave productivo agrícola inserto en las grandes cadenas agroalimentarias globales (De Castro et al. 2014; Moraes et al. 2012; Pedreño 2014). Este territorio ha tenido históricamente un débil desarrollo industrial, lo que ha reducido las oportunidades laborales formales, por lo que las clases trabajadoras se han ido conformando como figuras laborales hiperflexibles (Capecchi y Pesce, 1984), capaces de movilizarse para ocupar cualquier nicho laboral disponible, principalmente agricultura, construcción y turismo (Gadea et al. 2014). Se trata de un territorio donde la eventualidad es un rasgo básico de las relaciones laborales, existe una bolsa de trabajo informal incuantificable y unas normatividad moral presente en la vida cotidiana y en el trabajo. Estas características hacen insuficiente una aproximación cuantitativa.

La opción fue plantear una metodología cualitativa conceptual-inductiva que complementamos con metodología configuracionista (De la Garza, 2018). Esto nos permitió ser más flexibles para incorporar subjetividades y otros aspectos morales de la organización del trabajo y la vida. La técnica principal fue la realización de 42 entrevistas en profundidad guionizadas y organizadas según cinco perfiles que combiné diversas inmersiones etnográficas (una de ellas de un mes de estancia) y observación permanente de la vida cotidiana en el territorio de investigación. En este artículo propongo, a partir de la experiencia empírica de la investigación realizada, discutir la importancia crucial de la cuestión de la 
construcción de vínculos de confianza social en el territorio para desarrollar exitosamente una investigación sociológica cualitativa en un espacio local concreto.

\title{
PALABRAS CLAVE
}

Confianza; etnografía; construcción social; cualitativa; territorio

\begin{abstract}
The localities of Abarán, Blanca and Cieza form an important enclave of agricultural within the global agrifood chains fresh fruits (De Castro et al. 2014; Moraes et al 2012; Pedreño 2014). Historically, this territory has had a weak industrial development, which has reduced the formal labour opportunities, so the working classes have been becoming hyper-flexible labour figures (Capecchi and Pesce, 1984), capable of mobilizing to occupy any available labour niche, mainly agriculture, construction and tourism (Gadea et al. 2014). In short, we are planning to carry out a sociological investigation prioritizing the territorial entrance, in a space where the main characteristic is that the official statistics are insufficient to quantitative approach because the labour relations are characterized by eventuality as and exist informal work.

With this territory and his social organization, the option was to propose a qualitative conceptual-inductive methodology that we complemented with a "configurationist" methodology (De la Garza 2018) that allowed us to be more flexible to incorporate subjectivities and others aspects like morality aspects of the organization work process and life. The main technique was the in-depth, I did 42 scripted interview, organized according to five profiles, which combined various ethnographic immersions (one of which was a one-month stay) and permanent observation of daily life.

In this article I propose, based on the empirical experience of the research carried out, to discuss the crucial importance of the question of social building of trust in a qualitative research in a local territory.
\end{abstract}

\section{KEY WORDS}

Trust; ethnography; social building; qualitative research; territory. 


\section{INTRODUCCIÓN}

El presente artículo es producto de mi investigación de tesis realizada en la Vega Alta del rio Segura en la Región de Murcia ${ }^{1}$, un territorio cuyo modelo productivo agroeconómico está totalmente insertado en las cadenas globales agroalimentarias de productos en fresco (De Castro et al. 2017; Pedreño 2014). Se trata de un enclave productivo que se especializó en sectores de bajo valor añadido, intensivos en mano de obra, dependientes de mercados externos y marcados por la eventualidad (Capecchi y Pesce, 1984; Capecchi, 1991; Gadea, Ramírez y Sánchez, 2014; Pedreño et al. 2014). La relación temporal discontinua entre tiempos de trabajo formal y tiempo de desempleo, condiciona las estrategias de reproducción social de las familias de clase trabajadora del enclave, que Bourdieu define como "un sistema, un conjunto de estrategias particulares orientadas a la acumulación de distintos tipos de capital, por las cuales la familia tiende a reproducirse biológica y, sobre todo, socialmente, es decir, a reproducir las propiedades que le permitan mantener su posición" (2000:75).

En este sentido y debido a sus especiales características, este territorio y sus gentes "constituyen un excelente marco geográfico para reflexionar sobre los procesos constituyentes de un escenario rural característico de la realidad socioeconómica murciana (...) [que] se define por la diferenciación de sus mercados de trabajo e igualmente por la heterogeneidad de las estrategias sociales de subsistencia" (Pedreño, 1998:159). La Vega Alta ha atraído la atención de investigadores de todas las disciplinas en Ciencias Sociales. Es el caso de geógrafos como García Manrique que, ya en 1975, afirmó sobre la comarca que "una proporción elevada de la población, ni posee tierras, ni se dedica a la agricultura, aunque ésta esté presente en todo momento, pues la industria y los servicios -el transporte- son fundamentalmente de transformación y traslado de los productos agrícolas. Esto le da un aspecto de paisaje rural industrializado que merece una atención particular" (García, 1975:276). Otro geógrafo, José Luís González, apuntaba la particularidad de las regiones del sur con respecto a las del norte porque "el rasgo más destacado de la actividad económica de la comarca de la Vega Alta del Segura, poco frecuente en el conjunto del Estado español pero normal en las huertas levantinas, es la simbiosis agricultura-industria-servicios" (González, 1999:281). A la antropología también le han interesado las relaciones de producción y poder de estos territorios. Conocidos son los trabajos del catalán Joan Frigolé $(1982,1998)$ sobre las relaciones de patronazgo y los conflictos sociolaborales en la Vega Alta del Segura. También se han interesado historiadores, abogados y economistas relevantes como Miguel Rodríguez Llopis (2008), María Teresa Pérez Picazo (1984) Miguel Martínez Carrión (2002) y Mariano Ruiz-Funes(1916/1983). Desde la sociología también se ha optado por este territorio para comprender las lógicas organizacionales de los mercados de trabajo del sur de Europa y las estrategias de reproducción social de las clases

${ }^{1}$ Si bien entendemos el territorio como un lugar abierto a flujos de movilidad, sobre todo laborales, nuestro trabajo de campo se centró en los municipios de Abarán, Blanca y Cieza. 
populares (Gadea et al. 2014; De Castro et al. 2014; Moraes y Cutillas 2014; Moraes et al. 2012; Pedreño 1998, 1999, 2001,2010, 2014). Toda esta vasta producción científica nos dibuja un territorio caracterizado por relaciones laborales condicionadas por la eventualidad, donde la organización del trabajo formal ha estado históricamente controlada por una élite local, conformada por grandes empresarios agroindustriales y políticos locales y regionales, que han construido redes de poder clientelares con el doble objetivo de mantener su posición de poder de clase y controlar el mercado de trabajo.

Cómo puede dilucidarse, la elección territorial fue central en la construcción del objeto de estudio, lo que tuvo un peso importante en la concepción, planificación y desarrollo de la investigación. La priorización investigadora de la entrada territorial se debió a que este espacio es un laboratorio privilegiado para tratar de desentrañar la transformación de los mercados de trabajo del sur del sur de Europa (De Barros, 1992; Pugliese, 1991).

Mi investigación tenía como principal objetivo tratar de comprender como la normatividad moral y la organización social del trabajo, afectaba a la vida cotidiana de las clases populares trabajadoras en el marco del capitalismo flexible, la agricultura globalizada y la eventualidad. La investigación me planteaba dos estimulantes retos científicos. No me interesaba tanto el análisis objetivo y cuantificable de la realidad local. Mi intención investigadora fundamental era "establecer la relación entre el tipo de producción y las condiciones de trabajo, y entre las condiciones de trabajo y las condiciones de vida" (Grignon y Passeron 1992:145), para lo que no iba a ser suficiente con una aproximación cuantitativa. Era necesario ir "más allá" para tratar de hacer emerger lo invisible.

En este contexto, las clases populares parecían legitimar moralmente, con sus prácticas cotidianas y laborales, un modelo de organización social que les condenaba a desplegar complejas estrategias de reproducción social e inserción laboral con el objetivo de obtener rentas suficientes para la supervivencia. Para abordar esto utilicé el paradigma metodológico que propuso Enzo Mingione (1993), que defiende que la reproducción social de las familias de clase trabajadora no depende solamente de su inserción formal en el mercado de trabajo, sino de la capacidad que tienen cada uno de los miembros de la unidad familiar de desarrollar estrategias para satisfacer sus necesidades de subsistencia según: la clase que ocupan en la estructura social, las oportunidades que brinde el mercado de trabajo formal y las redes de reciprocidad, redistribución y autoabastecimiento.

Todo esto me llevó a resignificar la crisis económica de 2007 como una crisis de reconocimiento. La crisis estaba erosionando los consensos sociales que se habían ido construyendo durante décadas generando heridas morales en las clases populares. La mejor vía para conseguir mis objetivos fue proponer una investigación cualitativa, anclada en lo territorial, que nos permitiera recorrer los intersticios de las distintas esferas económicas, laborales, sociales y morales que componían las vidas cotidianas de las clases populares de la Vega Alta del Río Segura. Este artículo es producto de las reflexiones metodológicas que surgieron durante el proceso mismo de investigación. Tuve que ser flexible e imaginativo 
en mis prácticas para, a partir de una entrada territorial y etnográfica, ser capaz de construir vínculos de confianza con la población objeto de estudio, fundamentales para obtener la información necesaria.

\section{2. ¿CÓMO INVESTIGAR LO LOCAL? LA IMPORTANCIA DEL TERRITORIO, LA PLANIFICACIÓN Y LA COMPLEMENTARIEDAD METODOLÓGICA}

Antes de iniciar mis inmersiones etnográficas me plantee algunas preguntas genéricas que guiarían mis primeros pasos en el territorio. Quería saber si existía un lugar donde inmigrantes esperaran a primera hora de la mañana para ser reclutados para ir a trabajar. Si existía, las preguntas se dispararían en mi mente: ¿cuál era este lugar?, ¿a qué hora se reunían?, ¿quiénes eran?, ¿de dónde procedían?, ¿se conocerían entre sí?, ¿por quién eran reclutados?, ¿cuánto cobrarían?, ¿cómo trabajarían?.

Otra cuestión que me interesaba indagar era dónde se socializarían las clases populares trabajadoras y cómo buscarían trabajo ¿en bares, peluquerías, centros sociales, parques, iglesias, mezquitas, parques?, ¿de qué hablarían?, ¿cuáles eran sus preocupaciones cotidianas?, ¿dónde y cómo buscaban trabajo?, ¿qué peso tendrían las redes familiares? Las otras cuestiones estaban relacionadas con el ocio ¿cómo se organizaban las fiestas populares?, ¿quién participaba y quién las organizaba?, ¿tendrían un marcado cariz religioso o no?, ¿cuál sería el papel de las mujeres en este aspecto?, ¿y el de los migrantes? ¿dónde iban las familias los domingos?. Con todas estas preguntas apuntadas en un cuaderno y anotadas mentalmente me dispuse a realizar inmersiones etnográficas con el objetivo de observar y participar en la vida cotidiana con el objetivo de ser capaz de construir relaciones de confianza en el territorio que me permitieran establecer vínculos de cierta solidez con informantes claves; conseguir entrevistas de los perfiles que había programada y, si era posible, lograr alojamiento para vivir una temporada en el territorio.

Una vez que tenía claras las características centrales del territorio y había definido paradigma teórico y objeto de estudio, me plantee cómo debía abordar la investigación y que técnicas serían los más idóneas para alcanzar mis objetivos. Con esta base decidí utilizar las fuentes estadísticas secundarias disponibles a nivel regional, local y europeo en materia de empleo, demografía y renta, como complementarias a mi investigación cualitativa. Un análisis cuantitativo no sería suficiente para comprender las vertientes del trabajo y la vida cotidiana. De hecho, múltiples investigaciones anteriores a ésta (Capecchi et al. 1988; C.E.S 
19962; C.E.S 2006³; Sardá y Ghesta 2014; Ybarra 1998), habían demostrado anteriormente que en esta comarca del sur de Europa existen prácticas de trabajo informal, no declarado a la Seguridad Social que, sin embargo, articulan de forma importante las estrategias de supervivencia, que por su propia naturaleza no se registran oficialmente.

Por tanto, era consciente de que no podría acceder fácilmente a los aspectos subjetivos que barnizaban las relaciones sociolaborales, ni a las formas en que se objetivaba la normatividad moral específica del territorio; las formas de reconocimiento y consenso social; ni el peso de la historia local en la materialización práctica de las relaciones de poder. En consecuencia, necesitaba, como afirmó Gramsci, entender que "el movimiento de la historia depende esencialmente de la conciencia que los actores sociales tienen y de su capacidad de acción y de lucha" (Bagnasco, 2014:21).

Estas cuestiones me llevaron a optar por un paradigma teórico que privilegiara el enfoque reproductivo; apostara por una sociología de la vida y tuviera en cuenta todos los aspectos económicos de la vida cotidiana que escapan a la regulación del trabajo formal y el paradigma del mercado (Mingione, 1993; Pahl, 1991). En este contexto particular las estrategias de reproducción están en el centro de la interacción compleja entre tres elementos centrales: tiempo-trabajoterritorio en el marco de un modelo económico flexible prototípico de las economías del sur de Europa, caracterizados por elevado número de trabajos informales, relaciones laborales clientelares, elevado paro y perversión del reclutamiento de mano de obra (Capecchi y Pesce, 1984; Hadjimichalis, y Papamichos,1990; Hadjimichalis y Vaiou, 1987; Mingione, 1991).

En cuanto a la elección del método, es sabido que en ciencia existen dos grandes paradigmas científicos: el hipotético-deductivo y el conceptual inductivo. En el método hipotético-deductivo las teorías aparecen como hipótesis creativas de la imaginación del científico que después se confrontan, tratando de justificarlas, con las predicciones observacionales (Audi 2004). Se trata de un método que en Ciencias Sociales está más vinculado con la investigación cuantitativa pues trata de predecir el comportamiento de casos individuales a partir de una muestra amplia.

Para mi investigación partí de un planteamiento conceptual-inductivo clásico. A partir de varias preguntas de investigación establecí una hipótesis, mis objetivos y las técnicas de investigación para alcanzarlos. A partir de mi muestra, que no sería representativa cuantitativamente pero si cualitativa, obtendría conclusiones que extrapolaría a toda una clase social que operaba en un territorio

${ }^{2}$ C.E.S es el Consejo Económico y social. El equipo de investigación estaba conformado por profesores de la Universidad de Murcia: José Colino Sueiras (Director); José Daniel Buendía Azorín; Antonio García Sánchez; José Luján Alcaraz; Juan Ortín García; Miguel Ángel Pérez de Perceval y Prudencio José Riquelme Perea.

${ }^{3}$ En esta ocasión el equipo de investigación estuvo conformado por: José Colino Sueiras (Director); Prudencio José Riquelme Perea; Andrés Pedreño Cánovas; José Luján Alcaraz e Idelfonso Méndez Martínez. 
concreto. Además tenía ciertas pretensiones inductivas ${ }^{4}$, esto es, predictivas en el sentido de que las conclusiones pudieran convertirse en herramientas intelectuales para ser utilizadas en otras investigaciones con parámetros similares. El método inductivo me permitía actuar como "cazador tras las huellas" (San Martín, 2018) de los hallazgos que iría encontrando durante la investigación.

Pero las metodologías del positivismo lógico (hipotético-deductivo o conceptual-inductiva) se me antojaron insuficientes porque necesitaba atender el carácter procesual y dinámico de la investigación. Abarcar la complejidad de los procesos sociales que quería investigar y, sobre todo, dar respuesta a los cambios que se podrían ir produciendo durante el proceso de investigación, puesto que mi objeto de investigación eran sujetos que "hacían cosas". Consecuencia de un progresivo ejercicio de reflexión y de autoreflexividad sobre el proceso de investigación y las condiciones materiales para realizarla, decidí completar la metodología clásica conceptual-inductiva con una epistemología crítica que atendiera a la construcción temporal, es decir, histórica, de la investigación. $\mathrm{O}$ dicho de otra manera, incorporé elementos de la propuesta metodológica de la "descripción articulada» (Zemelman, 1987) y la "metodología configuracional" (De la Garza, 2018) para tratar de asir mejor la investigación y dar cabida a todas mis preocupaciones científicas que difícilmente encajaban en una tesis orientada linealmente según una metodología clásica.

La epistemología crítica del configuracionismo bebe de las ideas de Marx del concreto-abstracto-concreto; de la heterogeneidad cultural de Gramsci, la sociogénesis de Norbert Elias ${ }^{5}$, las configuraciones temporales de las relaciones sociales de E.P. Thompson y la escuela crítica de Frankfurt, sobre todo el método materialista de la reconstrucción de la totalidad concreta de Theodor Adorno y la propuesta de "pensar en imágenes" de Walter Benjamin. Para Enrique De la Garza "la metodología configuracionista no es una receta, esta se debe adaptar al objeto, al desarrollo de la ciencia, al tiempo y al espacio. Es una guía, en parte metodológica, pero también ontológica, para construir conocimiento" (2018:351)

Una de las grandes cuestiones metodológicas ha sido, y es, la relación que se construye entre estructuras, subjetividades y acciones sociales. La estructuración de la sociedad en clases sociales, no determina las prácticas reales de la gente.

${ }^{4}$ En el diccionario filosófico de Robert Audi encontramos dos definiciones de "inducción": 1) en un sentido estricto, la inferencia de una generalización a partir de sus instancias; 2) en un sentido más amplio, cualquier inferencia ampliativa en la que la afirmación hecha en la conclusión va más allá de la que introducen las premisas. O dicho de otro modo el inductivismo es la tesis de que las hipótesis pueden ser respaldadas por su éxito predictivo en otros casos particulares. (Audi, 2004)

${ }^{5}$ Enrique De la Garza escribe en varias ocasiones en su libro sobre metodología que no está de acuerdo con el concepto de "figuración" que utiliza Norbert Elias. Elias utilizó especialmente el concepto de "figuraciones" para tratar de situar en el centro teorético de la sociología la cuestión de las interdependencias humanas (Elias, 2008). En nuestro análisis ambas perspectivas conviven porque ambas ponen la mirada en lo que realmente nos importa: la centralidad de las interdependencias y de las relaciones sociales para tratar de comprender la organización de sociedades concretas y su proceso de evolución (y también permanencia) histórica. Sirva esto como reconocimiento al pensamiento de Elias. 
Son las prácticas cotidianas las que tienen realmente dimensión de sentido en una estructura social, aunque sus significados se muevan en un espacio finito, condicionadas por los límites de la cultura, la subjetividad y las estructuras transubjetivas (De la Garza, 2018; Heller 1987). Los significados que adjudicamos como investigadores a las acciones de los agentes sociales funcionan como un conglomerado de subjetividad entendida como "configuración de códigos para dar sentido a la situación concreta [que provienen de la cultura] entendida como sentidos socialmente acumulados, que son históricos" (De la Garza 2018:357). Cultura y subjetividad son diferentes pero codependientes y necesarias para tratar de reconstruir los códigos de las acciones en situaciones concretas, códigos que podrían ser morales, cognitivos, emocionales y/o estéticos (Bourdieu 2012; De la Garza 2018; Gramsci 2009, 2016; Thompson 1995, 2012).

En consecuencia, consideré interesante hacer un hueco en nuestra perspectiva metodológica a lo que De la Garza llama las "relaciones débiles" y las "relaciones duras" entre conceptos teóricos. Las "relaciones conceptuales duras" están conformadas básicamente por la lógica formal y el razonamiento. Las conceptualizaciones que se desprenden de las "relaciones débiles" incluyen las esquematizaciones; el papel de los sentimientos; de las creencias; las asimilaciones; los reenganches; mimetismos y, sobre todo, "el papel de la argumentación como vinculante conceptual" (De la Garza, 2018:194).

Esta forma de vehicular la metodología investigadora me abrió puertas para incorporar al análisis las intuiciones y percepciones que iban apareciendo durante el trabajo de campo. Lo que me permitió readaptar y reincorporar la experiencia personal que iba adquiriendo durante el mismo proceso de investigación. En definitiva, realicé una apuesta metodológica que pretendía flexibilizar su diseño para ser capaz de intuir e incorporar informaciones y materiales que me facilitaran acercarme mejor al fenómeno estudiado (Ruíz-Herrero, 2016).

\section{3. ¿CÓMO HACER LA INVESTIGACIÓN? LA IMPORTANCIA DE LAS TÉCNICAS.}

En toda investigación es fundamental elegir correctamente los métodos y técnicas para alcanzar los objetivos que nos proponemos y corroborar (o desechar) la hipótesis de trabajo, pero esta elección no debe ser puramente teórica, ni tener sólo en cuenta el objeto de estudio, sino también las condiciones de posibilidad del investigador pues "la fuerza metodológica de un instrumento no es sino aquella con la que puede contar epistemológicamente quien lo utiliza" (Grignon y Passeron, 1992:61).

En este caso, había diseñado una investigación sustentada en técnicas cualitativas (Bericat, 1998; Castro Nogueira y Castro Nogueira, 2001; García Jorba, 2000; Guasch, 2002; Vallés 1999, 2007) que combinaba el paradigma conceptual-inductivo con el paradigma configuracionista (De la Garza, 2018; Elias, 2008) que me permitiera desarrollar la investigación de un modo científico pero que al mismo tiempo fuese lo suficientemente flexible (Ruíz-Herrero, 2016) 
como para captar y abordar todos los aspectos que pudieran ayudarme a levantar la máscara fetichista que oculta la verdadera realidad subyacente (Harvey, 2014; Marx, 2014).

Para ello, era claro que tenía que sumergirme atentamente en el territorio, pasar tiempo en él. Ser capaz de hacer hablar a la gente y de escucharles, para lo que era crucial ser hábil en la construcción de alianzas y confianzas con la población objeto para acceder a sus discursos y prácticas cotidianas. No eran las personas investigadas las que debían adaptarse a mí, sino yo a ellas, entendiendo que habría posibilidades de que entendiéramos de diferente forma qué era justo/injusto o moral/inmoral. Si quería entender la particularidad subjetiva de las formas de vida de las clases populares trabajadoras en la Vega Alta debía insertarme en el territorio, tejer confianzas y adaptarme a sus códigos morales, motivaciones, juicios y valores.

Para que la gente hablara conmigo, primero habría que hablar con la gente. Acercarse, entablar conversación, saber qué decir, saber qué quería saber, qué palabras utilizar y de qué manera acercarme a una realidad que, en parte, me parecía desconocida y en parte me resultaba familiar por su similitud al lugar donde crecí. Se abría así una oportunidad excitante para tratar de comprender sin juzgar y acceder a las realidades ocultas de la vida cotidiana de las clases populares teniendo presente que son sujetos activos. Tal y como afirmó John Dunn:

Si queremos comprender a otras personas y postular la afirmación de que, de hecho, hemos conseguido comprenderlas, es imprudente y vulgar no hacer caso de lo que esas otras personas tienen que decir (...) lo que en propiedad no podemos hacer es afirmar que sabemos que lo comprendemos [al agente], o que comprendemos su acción mejor de lo que puede hacer él mismo si no tenemos acceso a las mejores descripciones que él pueda ofrecer" (Dunn 1980, citado en Scott 2013:24).

La entrevista en profundidad guionizada fue la técnica principal que elegí para acercarme a las "relaciones duras" y las inmersiones etnográficas en el territorio y la observación participante de la vida cotidiana para las "relaciones débiles" (De la Garza, 2018). El método y las técnicas no pueden estar separadas de las investigaciones concretas, deben estar ligadas a experiencias personales y ejemplos reales (Bourdieu, 2003; Bourdieu et al. 2005; Durand 2015; Guasch 2002; Wrigth 2010) en este caso mis intereses de investigación, las condiciones de posibilidad y el objeto de estudio.

Al comenzar la investigación se concretaron cinco perfiles de sujetos a entrevistar seleccionados según criterios de género; ocupación laboral; edad y nacionalidad. Para determinar el perfil de los entrevistados fue central el sentido de la investigación que priorizaba la entrada territorial al sectorial:

Perfil 1: Familias e individuos. Trabajadores y trabajadoras temporales y fijos- discontinuos, nacionales o inmigrantes ${ }^{6}$, de ambos sexos, con inserción laboral eventual o temporal.

${ }^{6}$ La inserción subalterna de los y las trabajadores migrantes en el modelo productivo de la 
Perfil 2: Instituciones públicas y/o sociales. Servicios Sociales, oficinas de empleo y organizaciones sociales.

Perfil 3: Grandes y medianos empresarios y productores agrícolas. Representantes políticos y sindicales.

Perfil 4: Informantes claves conocedores de la historia del territorio.

Perfil 5: Profesionales cualificados y pequeños empresarios locales de diversos segmentos.

Con estos perfiles buscábamos llegar al punto de saturación de los discursos. Dotando de sentido y coherencia, dentro de un mismo marco de análisis teórico, los relatos de las condiciones de trabajo y estrategias de vida económica de las clases populares. Para cubrir este objetivo de investigación realicé 42 entrevistas. Las transcripciones y las notas fueron objeto de una atenta lectura analítica, temática y comprensiva, buscando hacer emerger "las pautas estructurales que subyacen a un determinado conjunto de procesos sociales" (Bertaux y Bertaux, 1983:250)

Las visitas etnográficas y la observación de la vida cotidiana fueron continuas durante la investigación porque el territorio está a una distancia de unos 40 kilómetros de la ciudad de Murcia, donde habito. Existen muy buenas comunicaciones y accesos entre ambos territorios por la autovía A-30 dirección Madrid, siendo posible realizar el trayecto en unos 30 minutos en vehículo privado. Esto ha permitido que las visitas al territorio hayan sido continuas durante todo el proceso de investigación.

Estaba convencido de la importancia de estas inmersiones porque era la manera de tratar de percibir los discursos y las prácticas cotidianas que desbordan el control hegemónico, lo que me daría claves de las formas de vivir, resistir y el modo en que desarrollan sus prácticas morales las clases populares en un territorio concreto. El poder pretende siempre penetrar en los espacios de sociabilidad popular para moldearlos según sus intereses, pero estos espacios son defendidos por las clases subalternas mediante incentivos y sanciones sociales, creando barreras invisibles pero existentes entre el "nosotros" y el "ellos". Son lugares donde las clases populares se expresan más libremente (Thompson, 2012) incluyendo "discursos ocultos" (Scott, 2003). Estos lugares de sociabilidad popular van variando con el tiempo pero algunos se mantienen como centrales como el bar, la calle y las asociaciones y eventos sociales como las fiestas populares y los encuentros familiares. Pero había un problema, y es que yo era un extraño que, además, venía de la Universidad.

\footnotetext{
Vega Alta es una de las claves para entender la organización del trabajo (Avallone y Ramírez,
} 2017) 


\section{4. ¿HABLANDO CON LA GENTE. LA IMPORTANCIA DE LA CONFIANZA PARA CONSEGUIR ENTREVISTAS.}

La entrada como investigador en un territorio rural no es sencilla. La desconfianza por mi vinculación a la Universidad estaba latente como elemento perturbador de la vida cotidiana de la población. En primer lugar, era un desconocido para las personas a las que quería investigar y la desconfianza hacia lo institucional es un rasgo característico de las clases populares, bien por desconocimiento bien porque crean tener motivos para ocultar cualquier aspecto de sus vidas a personas vinculadas a estamentos públicos.

Como investigador tenía objetivos que cumplir, preguntas en la cabeza y respuestas que quería encontrar, pero es difícil poder controlar todos los aspectos y saber de antemano cuáles son las expectativas (creadas o asumidas) que la contactación genera en la futura persona entrevistada, porque en ocasiones los entrevistados también tenían expectativas sobre el investigador y la investigación, muchas no verbalizadas o expresadas directamente ${ }^{7}$.

Por ejemplo, aquel trabajador autónomo dueño de una peluquería local que veía en mí a "alguien" del Ministerio o de la Comunidad, un interlocutor político-administrativo válido al que contar los problemas con que se encontraba en su quehacer diario. La entrevista fue compleja porque sólo quería hablar de su situación socioeconómica y de la competencia desleal. Esto me obligó a tener paciencia y a escuchar para poder reorientar la entrevista en cuanto fuese posible. Al final fue una entrevista muy interesante porque conseguimos algunas claves de las actividades ilegales que le hacían competencia, es decir, de estrategias de supervivencia. Otro tanto ocurrió con una trabajadora inmigrante, con una dura realidad vital presente y un pasado aún más complicado. A punto de ser desahuciada, pérdidas sus raíces en su país de origen, engañada y abandonada por el que era su compañero, con sus hijos dispersos y sin trabajo, buscó en mí un apoyo para contarme su relato, su verdad de su vida. Precisamente, tratar de mejorar la vida cotidiana de las personas con las que tratamos es uno de los ambiciosos objetivos más difíciles de alcanzar, pues es sabido que el trabajo científico es diferente a la voluntad política.

Romper desconfianzas era el primer paso para poder acceder a los perfiles que me interesaba. Para ello fue necesario desarrollar relaciones de confianza y cooperación en el terreno donde la construcción social de la confianza fue un asunto crucial (Equipo Enclaves, 2016; Guasch, 2002).

En mi caso contaba con una herramienta para romper esa desconfianza. Estuve trabajando en la agricultura, primero en el campo durante los veranos desde los 15 años, luego dos temporadas de un año completo en dos almacenes de envasado en dos empresas diferentes. Trabajé en todos las tareas que completan un ciclo productivo. Este bagaje personal me sirvió cuando la investigación me retrotraía a mi propia experiencia personal, porque me permitía recodificar ciertos lenguajes y posiciones sociales, y creo, firmemente, que comprenderlos me-

\footnotetext{
${ }^{7}$ Para profundizar en los encuentros etnográficos de mi investigación véase Ramírez (2019)
} 
jor. Como defendía Wrigth Mills en su mítica obra "la imaginación sociológica" nuestra experiencia de vida influye en nuestro trabajo presente porque prestamos valor a la experiencia vital directa (Wright, 2010). Sin duda, estas experiencias vitales propias tuvieron incidencia en la elección de mi tema de estudio, porque tal y como afirmaba Pierre Bourdieu (2003) el investigador puede y debe movilizar su experiencia, su pasado, en todas las fases de la investigación.

Dominar cierto vocabulario específico relacionado con el proceso de trabajo, la maquinaria y la organización de los modos de producción me facilitó que algunos entrevistados, sobre todo de las clases populares, se sintieran más cómodos en las entrevistas. Conocía las diferentes tipologías de personas que trabajan en un almacén, como se organizaba toda la cadena de trabajo. Cuando trabajé sufrí las largas y duras jornadas laborales en temporada alta, el no descansar, los olores que desprenden los frutos cuando son tratados, las malas formas en que se trataba a la gente mientras trabajaban. Trataba que la persona que estaba entrevistando supiera que era empático con ella porque había sido "uno de ellos" no por pena ni nada parecido. Para ello trataba de entablar conversación antes de encender la grabadora, cuando no era posible, utilizaba este bagaje durante las entrevistas. Todo esto generaba confianza porque reequilibraba simbólicamente la diferencia social que los entrevistados sentían al sentarse frente a "profesores de la universidad". Diferencia social que por lo general desaparecía tras unos minutos de conversación. Al principio no fui muy consciente de ello porque surgía de un modo natural, pero con el análisis de las entrevistas pudimos darnos cuenta de la importancia que la construcción de este tipo de vínculos estaba teniendo para conseguir entrevistados y desarrollar exitosamente la investigación.

Otro aspecto que me ayudo es que no parezco un profesor universitario, ni siquiera alguien vinculado con la Universidad o con estudios superiores. Mi forma de hablar, de estar y de vestir facilitó que la gente se identificara fácilmente conmigo y me identificaran como miembro de su misma clase social, con sus códigos y lenguajes... aunque en realidad no sea (ya) del todo así.

Una de las cuestiones donde la confianza fue central, fue la contactación. Dos han sido las principales vías a la hora de conseguir entrevistados. Para perfiles técnicos y profesionales realizamos una entrada formal, contactando directamente con la organización, empresa o entidad pública donde desarrollaban su labor. Para perfiles más sociales los contactos se conseguían yendo al terreno, pisando calle y construyendo vínculos con la gente del pueblo que, aunque tuviera claro las diferentes posiciones que ocupábamos en la escala social, no impidiera la comunicación lo más sincera y abierta posible.

Para el segundo grupo (más social e informal), seguí tres protocolos diferentes: buscaba directamente en el territorio los perfiles determinados en la muestra; usaba la técnica de "bola de nieve" a partir de las entrevistas realizadas, y en tercer lugar, por medio de personas que por su posición social dentro de la localidad actuaron de porteros abriéndonos o cerrándonos, según el caso, el acceso a sujetos muestrales interesantes. En este caso el modus operandi era que los/ las portero/as hablaban con los candidatos y tras su consentimiento los porterosenlaces nos facilitaban el teléfono del candidato/a para concertar una cita porque 
"es la proximidad al fenómeno investigado lo que facilita el acceso al campo y a los escenarios" (Guasch, 2012:37). Realizamos entrevistas en lugares tan diversos como bares, sedes de sindicatos, viviendas particulares, despacho de la universidad, oficinas de empresas e incluso dentro de un vehículo mientras recorríamos el territorio. La duración media de las entrevistas osciló entre una hora y una hora y media, llegando algunas a más de dos horas.

\section{LA IMPORTANCIA DE LA CONFIANZA EN LAS INMERSIONES ETNOGRÁFICAS Y LA OBSERVACIÓN DE LA VIDA COTIDIANA}

Para la observación participante de la vida cotidiana adopté una metodología clásica, producto de las aportaciones del interaccionismo simbólico de Goffman (1970), la etnomedotología de Garfinkel (2006) y la Escuela de Chicago, en la que la etnografía actúo como una forma de investigar la vida cotidiana. Existen diferentes modos de observar en Ciencias Sociales, nuestro método de observación escogido fue ser participante ordinario en la sociedad que analizaba (Vallés, 1999), esto es, cuando se trataba de la vida cotidiana, del ocio, la festividad o las vivencias en la calle, adoptaba el rol de observador-como-participante (Vallés, 1999:153). Hacer observación participante durante mi estancia etnográfica me permitió obtener varias ventajas notables. Me dio la oportunidad de forjar eslabones en mi cadena de confianzas y me permitió pasar desapercibido en los espacios públicos sin alterar con mi presencia a los agentes sociales.

A comienzos del verano de 2013, tras meses de hacer entrevistas y establecer lazos de confianza con la población local, surgió la oportunidad de pasar una temporada en Cieza, el principal municipio de nuestro territorio de investigación. Gracias a la generosidad de la familia Villa-Abellán pude disponer de una vivienda gratuita y acondicionada en el casco urbano del municipio, cerca de un punto de recorrida de jornaleros cada mañana. Permanecí viviendo en el municipio durante 23 días, desde finales de agosto a principios de Septiembre del 2013. Esta favorable circunstancia me brindó la oportunidad de contactar con gentes in situ, realizar un buen número de entrevistas, acudir por las mañanas a los lugares de reclutamiento de los trabajadores inmigrantes, pasear por sus calles y plazas observando la modelación del paisaje, las industrias abandonadas, los pequeños comercios, almacenes, cooperativas, los barrios obreros. Así mismo me permitió impregnarme del terreno, conversar informalmente con sus gentes, visitar el museo del esparto con asiduidad y entablar amistad con socios (la mayoría de ellos ex trabajadores de las clases populares), consultar el archivo municipal y participar en sus fiestas patronales locales que se celebraron la primera semana de Septiembre.

De todo ello surgió un diario de campo que ayudó a entender mejor el objeto de estudio porque el devenir de la investigación me obligó a un proceso de reflexividad permanente sobre mi trabajo y los pasos que iba dando en la investigación. De esta manera fueron surgiendo situaciones no controladas pero que sin embargo fueron muy interesantes para la investigación pues me ofrecieron la 
posibilidad de explorar lo oculto, de adentrarme en zonas y territorios(físicos y subjetivos) que no había previsto previamente, a insértame en las redes locales y a ir tejiendo y retejiendo el trabajo de campo.

En ocasiones mi capacidad de no parecer un profesor universitario se transformó en debilidad. Ocurría cuando los sujetos entrevistables desconfiaban de mi posición académica, fue ahí cuando detecté la necesidad de poseer identificación de mi vinculación con la Universidad de Murcia, como parte de la construcción de la confianza. Cuando existía desconfianza hacia mi rol de investigador el trabajo de campo fue más complicado y surgía la necesidad de "ganarme la confianza" en el terreno o con alguna otra herramienta.

Esto me ocurrió cuando traté de contactar con trabajadores inmigrantes marroquíes que se apostan cada mañana en un lugar concreto para ir a trabajar. A priori la información con la contaba, era que había un lugar concreto, cerca de un supermercado, donde los jornaleros marroquíes esperaban para ser recogidos y llevados a trabajar al campo. Los informadores de esta situación eran vecinos del pueblo, nunca habían esperado en ese punto ni sabían a ciencia cierta si eran legales o ilegales, contratados o informales. ¿Cómo abordar la contactación? Existen diferencias notables entre si son legales o no por ejemplo ¿Cómo averiguarlo? Ciertamente no teníamos ningún contacto marroquí, no teníamos enlaces sindicales ni un "portero" que nos facilitar la entrada en el campo. Había que intentarlo directamente pero ¿cómo?.

La forma de acercarse a un grupo de trabajadores inmigrantes que esperan al alba en una calle no es sencilla porque está llena de incertidumbres. Algunos días, a primera hora de la mañana pasé en coche por las calles tratando de concretar el punto donde se encontraban. Una vez hecho esto, advertí que los migrantes esperaban en diferentes lugares para ser recogidos y llevados al tajo. No estaban todos en el mismo lugar, ni formaban grupos homogéneos, ni llevaban los mismos utensilios de trabajo ni se relacionaban entre ellos de la misma manera, aunque todos estaban en un área cercana entre sí. Se agrupaban de dos maneras diferentes, por un lado había un gran grupo de unos 25-30 hombres marroquíes juntos en un mismo lugar. Por otro lado había pequeños grupos desperdigados de no más de 4 o 5 hombres o incluso solitarios en los alrededores de donde se concentraba el gran grupo. La hipótesis, totalmente incomprobable para el investigador en ese momento, era que la zona donde estaba el grupo principal era el lugar se recogían a los inmigrantes reclutados más o menos formalmente para ir a trabajar. Se trataría de jornaleros legales que eran citados cada mañana en ese lugar para ser transportados en furgonetas. En consecuencia no tenían que esperar a ser recogidos ni competir con otros jornaleros pues ya eran reclutados ¿de qué manera? El resto de grupos pequeños más desperdigados podían ser trabajadores legalizados o no, que no habían conseguido ser reclutados por las redes informales de contactación, que en este caso ejemplifican los "furgoneteros". El segundo día que visité el lugar de recogida lo hice durante más tiempo para comprobar cómo los hombres que esperaban agrupados marchaban al alba a trabajar juntos. A las 9 de la mañana aún habían grupos disper- 
sos esperando a que alguien los reclutara para "echar el jornal", pero a esas horas podemos afirmar que ya no trabajarían.

El tercer día había que intentar contactar con los trabajadores del gran grupo pero ¿con qué argumentos? ¿de qué manera? ¿cómo se acerca un desconocido, de otra etnia, a un grupo de hombres migrantes desconfiados que esperan al raso para ir al campo a trabajar durante una dura jornada?

Cuando traté de entablar conversación para conseguir contactos que más debieran ser entrevistas, lo hice de un modo torpe pero efectivo. Los trabajadores migrantes habían sospechado de mí porque me habían visto los días previos merodear con mi vehículo. Así que fueron los inmigrantes los que me pararon de forma expeditiva cuando pasé conduciendo delante de ellos. Varios me bloquearon el camino poniéndose delante del coche. No fue necesario que me dirigiera a ellos, puesto que fueron los jornaleros los que, desde la desconfianza y el temor, me pararon para pedirme explicaciones por mi comportamiento y para saber mi identidad. En ese instante sólo tuve unos pocos minutos para reaccionar y utilizar las herramientas con los que cuenta un investigador, tratar de ser honesto y explicar rápidamente qué eres y qué quieres. Tras unos minutos de incomprensión mutua conseguí contactar con el portavoz del grupo, el único que hablaba castellano. Me pidió el teléfono por si "había problemas" pues pensaban que era un policía o un inspector de trabajo. Le di mi teléfono y le dije que me llamara en el momento para que comprobara que no le estaba engañando. Me contestó que no tenía saldo. En ese momento reaccioné pidiéndole su número de teléfono con la excusa de dejarle una llamada perdida y que retuviera mi teléfono, así podrían confiar en mí... y yo tendría su teléfono.

Esa misma tarde lo llamé. Le pedí ir a entrevistarlo a su lugar de residencia pero se negó porque le podría traer problemas con otros compatriotas y compañeros de trabajo que me habían visto aquella mañana. Entonces le invité a tomar café a casa, pareció sorprenderse pero accedió y concertamos una entrevista en la vivienda que me habían facilitado, a la que accedió gustoso. Estuvimos charlando casi dos horas, aunque no me dejó grabar la conversación. Tomé muchas notas al marcharse obteniendo una valiosa información, además de sentirme reconfortado al tener la oportunidad de pedirle perdón por haberles asustado aquella madrugada. Al despedirnos le regalé algunos libros de compañeros sociólogos para que supiera lo que se escribe sobre ellos. En esta ocasión la confianza se construyó en un primer momento siendo honesto y posteriormente ofreciéndole un lugar donde pudiera contar su dura historia de inmigración jornalera.

\section{ALGUNAS REFLEXIONES FINALES}

Durante el tiempo de investigación las personas entrevistadas me invitaron a entrar en sus vidas, sus casas, me mostraron sus cosechas, enseñaron fotos de sus familiares, me invitaron a almorzar y a beber construyendo vínculos de confianza que debían ser necesariamente efímeros pero honestos. El trabajo de campo tiene consecuencias, múltiples, complejas, que desbordan ese momento 
mágico de encuentro puntual con informantes. Encuentros construidos y buscados, a veces conseguidos tal y como los planificamos, otras de diferente manera a la prevista. La gente siempre tiene cosas que decir y los y las investigadores cuestiones que aprender. El respeto, la claridad de nuestros objetivos y el tratar de establecer un diálogo de escucha activa, no ya entre iguales, sino de subsunción del investigador a los discursos del investigado están en la base de conseguir entrevistas y encuentros, palabras y momentos. La gente necesita/quiere ser escuchada, contar su historia. Pero para hacerlo deben sentirse cómodas con sus interlocutores, debe construirse un clima relacional basado en la confianza mutua que permita que las personas entrevistadas se expresen con las menos barreras posibles. En este sentido, una de las capacidades fundamentales de cualquier persona que investigue lo social, una de las claves del oficio de sociólogo/a es saber construir vínculos de confianza.

\section{BIBLIOGRAFÍA}

Audi, R. (ed) (2004) Diccionario Akal de Filosofía. Akal. Madrid.

Avallone, G y Ramírez-Melgarejo A. J. (2017). Trabajo vivo, tecnología y agricultura en el Sur de Europa. Una comparación entre la Piana dele Sele en Salerno (Italia) y la Vega Alta del Segura en Murcia (España). AGER, Revista de Estudios sobre Despoblación y Desarrollo Rural n. ${ }^{\circ}$ 23, pp. 131-161. Recuperado de: http://www.ceddar. org/content/files/articulof 39907 Ager-23-05-Avalone-02.pdf

Bagnasco, A. (1991a). El desarrollo de economía difusa: punto de vista económico y punto de vista de la sociedad. Sociología del trabajo, nueva época. Número extraordinario, pp. 167-174.

(1991b). Desarrollo regional, sociedad local y economía difusa. En E., Reynaud. M., Maruani y C., Romani, (coords.) Debates sobre el empleo [1] en Italia, pp. 331-342. Madrid: Ministerio de Trabajo y Seguridad Social

------. (2007) "El capitalismo que cambia, el trabajo y las condiciones de vida" Sociología del trabajo, nueva época. Madrid. no 61. Pp. 7-29.

----. (2014) Gramsci y la sociología. Sociología del Trabajo no 82. Pp 16-27

Bericat, Eduardo (1998). La legitimidad científica de la integración. La integración de los métodos cuantitativo y cualitativo en la investigación social. Significado y medida. Barcelona. Editorial Ariel.

Bertaux, D. y Bertaux, I. (1983). Historias de vida del oficio de panadero. California. $2^{\mathrm{a}}$ Edición. California: Biography and Society.

Bourdieu, P. (2012) La distinción Taurus. Madrid.

------- (2003) L'Objectivation participante. Actes de la Recherche en Sciences

Sociales. $\mathrm{N}^{\mathrm{o}} 150$. Pp. 43-58.

-----. (2000). Cuestiones de Sociología. Madrid: Akal.

Bourdieu, P., Chamboredon, J.C. y Passeron, J.C. (2005). El oficio de sociólogo. $5^{\mathrm{a}}$ Edición. Madrid: Siglo XXI.

Capecchi, V. (1991) Pequeña empresa y economías locales: la flexibilidad productiva. En E., Reynaud. M., Maruani y C., Romani, (coords.) Debates sobre el empleo [1] en Italia, pp. 311-330. Madrid: Ministerio de Trabajo y Seguridad Social

Capecchi, V. y Pesce, A. (1984). Si la diversidad es un valor. Debats nº10, pp. 29-49. 
Capecchi, V.; Sanchis, E. y Miñana, J. (1988) La otra economía: Trabajo negro y sector informal. Valencia. Alfons el Magnanim.

Castro, M. A. y Castro, L. (2001). Cuestiones de metodología cualitativa. Empiria Revista de Metodología de Ciencias Sociales, nº4, pp 165-190.

Consejo Económico y Social de la Región de Murcia (C.E.S) (2006) La economía sumergida en la Región de Murcia. CES. Colección de Estudios n²0. Murcia. ---------. (1996). Mercado de trabajo e irregularidades laborales en la Región de Murcia. CES. Colección de Estudios n². Murcia.

De Barros, A. (1992). Trabajo en el espacio social rural. Revista Estudios Regionales, ${ }^{\circ}$ 31, pp77-86.

De Castro, C. Gadea, E. y Pedreño, A. (2014). Inmigración, crisis del sur de Europa y sostenibilidad social de las estrategias de desarrollo. El caso de los enclaves productivos de agricultura intensiva. Revista Trabajo, tercera época, año 8, nº 11, pp. 89115. Recuperado de: http://www2.izt.uam.mx/sotraem/Documentos/Revistadetrabajo11final.pdf

De Castro, C.; Gadea, E. Pedreño, A. y Ramírez-Melgarejo, A. J. (2017). Coaliciones sociales y políticas en el desarrollo del sector agroexportador: las frutas murcianas en las redes globales de producción agroalimentaria. Revista Mundo Agrario. Argentina. Recuperado de: https://www.mundoagrario.unlp.edu.ar/article/view/MAe043

De la Garza, E. (2018) La metodología configuracionista para la investigación. México: Editorial Gedisa. Universidad Autónoma Metropolitana. Unidad Iztapalapa. Recuperado de: http://www2.izt.uam.mx/sotraem/NovedadesEditoriales/MetodologiaConfig SD.pdf

Dunn, J. (1980). Practising history and social science on 'realist' assumptions. En Political Obligation in its Historical Context: Essays in Political Theory, pp. 81-111. Cambridge: Cambridge University Press.

Elías, N. (2008) Sociología fundamental. Biblioteca Económica GEDISA. Segunda reimpresión. Barcelona

Equipo Enclaves-Universidad de Murcia (2016) “¿Cómo hicimos el proyecto de investigación enclaves: 'Sostenibilidad social de los enclaves de agricultura intensiva: España y México (2012-2015)?"' Sociología del trabajo, nueva época no86 pp. 107124. Madrid.

Frigolé, J. (1998) Un hombre: Género, clase y cultura en el relato de un trabajador. Barcelona. Editorial Muchnik. -------. (1982) "Aparcería y conflicto en un pueblo de la vega alta del segura (1962-1974)” Revista Áreas. Murcia. n². Pp. 72-81.

Gadea, E. Ramírez-Melgarejo, A. J. Sánchez, J. (2014). Estrategias de reproducción social y circulaciones migratorias de los trabajadores en los enclaves globales. En A. Pedreño (coord) De cadenas, migrantes y jornaleros: los territorios rurales en las cadenas globales agroalimentarias, pp. 134-150. Madrid: Talasa.

García Jorba, J.M. (2000) Diarios de campo. № 31 Cuadernos Metodológicos. Madrid: Centro de Investigaciones Sociológicas.

García Manrique, E. (1975). Un ejemplo de paisaje agrario de la Vega Alta del Segura: Abarán. Estudios Geográficos, vol36, n¹38-139, pp. 417-451.

Garfinkel, H. (2006). Estudios en Etnometodología. Barcelona: Anthropos.

Goffman, E. (2004). La presentación de la persona en la vida cotidiana Buenos Aires: Amorrotu.

González Ortiz, J. L. (1999) “Geografía de la Región de Murcia” Colección Monografías Regionales. $\mathrm{n}^{\circ} 3$ Murcia. Editorial Regional de Murcia. 
Gramsci, A. (2009) La política y el Estado moderno. Madrid. Biblioteca Pensamiento Crítico. Público.

(2016) Antología para la reforma moral e intelectual. Madrid: Catarata. Colección Clásicos del pensamiento crítico.

Grignon, C. y Passeron, J. C. (1992) Lo culto y lo popular. Madrid. La Piqueta.

Guasch, O. (2002). Observación participante. Madrid: Cuadernos metodológicos del CIS, $n^{\circ} 20$.

Hadjimichalis, C. y Vaiou, D. (1987) “La evolución del desarrollo desigual y formas de reproducción social en Grecia” Documents d'Análisis Geográfica Barcelona. n¹0. Pp5-23.

Hadjimichalis, C. y Papamichos, N. (1990). Desarrollo local en el sur de Europa: hacia una nueva mitología. Estudios Regionales, n²6, pp. 113-144.

Harvey, David (2014) Diecisiete contradicciones y fin del capitalismo Madrid. Traficantes de sueños.

Heller, A. (1987) Teoría de los sentimientos. Madrid. Fontanera.

Martínez, J. M. (2002) Historia económica de la Región de Murcia Colección Monografías Regionales ${ }^{\circ}$ 4. España. Editorial Regional de Murcia.

Marx, K. (2014) El capital: Crítica de la economía política, Libro I. El proceso de producción del capital. México: Fondo de Cultura Económica.

Mingione, E. (1993) Las sociedades fragmentadas: una sociología de la vida económica más allá del paradigma del mercado. Ministerio de Trabajo y Seguridad Social. Madrid

-------. (1991) Paro, subempleo, trabajo negro e informal en el sistema socio-económico del sur. Revista Estudios Regionales, n³1, pp. 173-194.

Moraes, N. y Cutillas, I. (2014). Nuevos dispositivos de regulación transnacional: un análisis sobre los estándares de calidad y responsabilidad social y su impacto en los enclaves globales agrícolas. En A. Pedreño (coord.) De cadenas, migrantes y jornaleros, pp. 195-219. Madrid: Talasa.

Moraes, N.; Gadea, E.; Pedreño, A. y De Castro, C. (2012). Enclaves globales agrícolas y migraciones de trabajo: convergencias globales y regulaciones transnacionales. Política y Sociedad, vol 49, nº 1 , pp. 13-34.

Pahl, R. (1991) Divisiones del trabajo. Madrid. Ministerio de Trabajo y Seguridad Social.

Pedreño, A (2014) De cadenas, migrantes y jornaleros: los territorios rurales en las cadenas globales agroalimentarias. Talasa. Madrid.

--------. (2010) Familias inmigrantes: el trabajo de los padres y las estrategias de trabajo de los hijos en las áreas mediterráneas de agricultura intensiva. En S.M. Lara (coord.) Migraciones de trabajo y movilidad territorial, pp. 333-367. México: Consejo Nacional de Ciencia y Tecnología.

-------. (2001) Efectos territoriales de la globalización: el caso de la ruralidad agroindustrial murciana. Revista de Estudios Regionales, 2a época. Enero-Abril, n ${ }^{\circ} 59$. pp. 69-96.

---.-. (1999) Del jornalero agrícola al obrero de las factorías vegetales. Madrid. Ministerio de agricultura, pesca y alimentación.

-------. (1998) Economía flexible y ruralidad: el caso de la Vega Media del río Segura en la Región de Murcia. En J.M. Ybarra (coord.).Economía sumergida: el estado de la cuestión en España, pp. 159-221. Murcia: U.G.T 
Pedreño, A.; Gadea, M.E y Latorre M. (2013). Nuevos y viejos conflictos jornaleros en Murcia, 1890-2012. Comunicación al Congreso nacional de Sociología de la F.E.S. España. Recuperado de: https://www.fes-sociologia.com/nuevos-y-viejos-conflictos-jornaleros-en-murcia-1890-2012/congress-papers/1708/

Pedreño, A.; De Castro, C.; Gadea, M.E; Moraes, N. (2015). Sustainability, resilience and agency in intensive agricultural enclaves. Ager: Revista de estudios sobre despoblación y desarrollo rural, $\mathrm{n}^{\circ} .18$, pp. 139-160.

Pérez Picazo, M.T y Lemeunier, G. (1984) El proceso de modernización de la región murciana (Siglos XVI-XIX). Murcia. Editora Regional de Murcia.

Pugliese, E. (1991). Dos Italias en dos Europas: Mercado de trabajo y estructura laboral en el Sur con referencia al marco europeo. Revista Estudios Regionales, n⿳3031, pp. 115-125.

Ramírez, A. J. (2019) Hacia una nueva cuestión meridional: crisis de reconocimiento y heridas morales en las clases populares de la Vega Alta del río Segura (Región de Murcia). Tesis doctoral. Universidad de Murcia. Recuperada de: http://hdl.handle. net/10201/77461

Rodríguez Llopis, M. (2008) Historia General de Murcia. España. Editorial Almuzara S.L. Tres Fronteras Ediciones.

Ruíz-Herrero, J. A. (2016). Propuestas para resolver dificultades en la investigación: Cómo activar materiales de análisis y otros recursos. Empiria. Revista de Metodología de Ciencias Sociales, n³4 mayo-agosto 2016, pp. 79-100.

San Martín, D. (2018). ¿Artesanía o cazador tras la huella? Reflexiones para el análisis cualitativo de datos. Empiria. Revista de Metodología de Ciencias Sociales, nº40 mayo-agosto 2018, pp. 65-83.

Sardá, J. y Técnicos de Ministerio de Hacienda (Gestha) (2014). La economía sumergida pasa factura. El avance del fraude en España durante la crisis. Lleida (Cataluña): Gestha y Fundación Universitat Rovira y Virgili.

Scott, J. C. (2013). Elogio del anarquismo. Barcelona: Crítica.

--.-. (2003). Los dominados y el arte de la resistencia. Navarra: Txalaparta.

Thompson, E. (2012). La formación de la clase obrera en Inglaterra. Madrid: Capitan Swing.

------. (1995) Costumbres en común. Crítica. Barcelona

Vallés Martínez, M. S. (2007) Entrevistas cualitativas. Madrid. № 32 Cuadernos Metodológicos. Centro de Investigaciones Sociológicas.

-------- (1999) Técnicas cualitativas de investigación social: reflexión metodológica y práctica profesional Madrid. Editorial Síntesis.

Wright, M. C (2010). La imaginación sociológica. Fondo de Cultura Económica. México.

Ybarra, J.A (1998) (ed) Economía sumergida: el Estado de la cuestión en España. Madrid. Unión General de Trabajadores (U.G.T)

Zemelman, Hugo (1987) Uso crítico de la teoría. México. Instituto Politécnico Nacional. 
